\title{
The Fast Track Trigger at the H1 Experiment Design Concepts and Algorithms
}

\author{
André Schöning \\ Institute for Particle Physics, ETH Hoenggerberg, CH-8093 Zürich
}

\begin{abstract}
The H1 Collaboration has built a new Fast Track Trigger which performs a full reconstruction of charged particles in a high multiplicity environment. At the first trigger level $(2.3 \mu \mathrm{s})$ coarse tracks are reconstructed in two dimensions whereas at the second level $(23 \mu \mathrm{s})$ all tracks are reconstructed with high precision in all three dimensions. This allows to identify particle resonances at the third trigger level ( $\lesssim 100 \mu \mathrm{s})$. The conceptual design and the implementation of the fast track finding algorithms are discussed. First results from the 2005 running period are presented.
\end{abstract}

\section{Introduction}

The H1 Collaboration at the ep collider HERA has built a new track based trigger system, the Fast Track Trigger (FTT). It is integrated in the first three levels (L1-L3) of the H1 trigger scheme and performs a track and event reconstruction with increasing precision at higher levels. At the first level it provides a fully pipelined (at $10 \mathrm{MHz}$ ) 2-dimensional reconstruction of tracks in the central drift chamber (CJC) down to transverse momenta of $100 \mathrm{MeV} / \mathrm{c}$ within a latency of $2.3 \mu \mathrm{s}$. At the second level a full 3-dimensional reconstruction of tracks is performed within $23 \mu$ s with a track resolution comparable to the full offline reconstruction. At the third trigger level event properties and resonant decays are reconstructed by computing invariant masses for all particle combinations. This allows to trigger on exclusive final states already at a very early stage $(\lesssim 100 \mu$ s)

The construction of the FTT started in 2000 when large size high density Field Programmable Gate Arrays (FPGA) with embedded Content Addressable Memories (CAM) became available. This made it possible to implement highly sophisticated reconstruction algorithms in hardware. High precision was achieved by implementing a non-iterative track fit in data signal processors (DSP). 


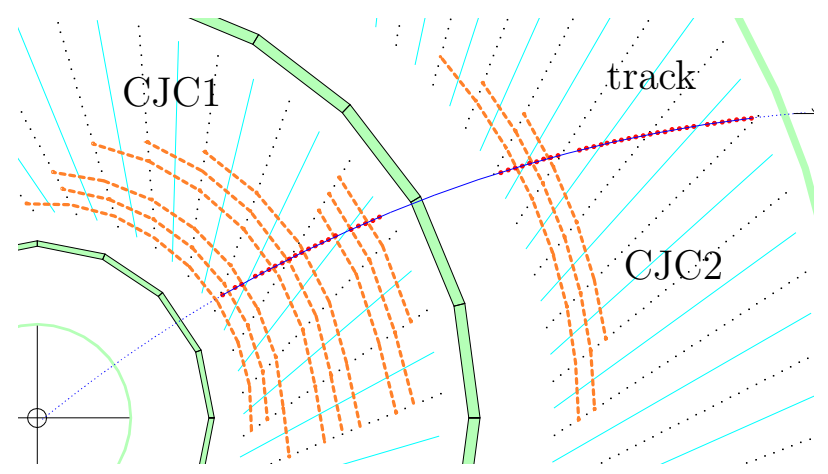

Fig. 1. Sketch of a track passing through the H1 Central Drift Chamber. The wire layers used by the FTT are marked.

\section{Fast Track Trigger Design}

The ambitious design goal, a fast and precise track reconstruction realized on hardware level together with tight timing requirements, poses several constraints on the system design.

In order to reduce costs and bandwidth only twelve out of the in total 56 axial layers of wires are used as trigger input, resulting in an input bandwidth of $72 \mathrm{Gbit} / \mathrm{s}$ using $80 \mathrm{MHz}$ ADCs. The twelve layers of wires are grouped in four so called trigger layers, each consisting of three wire layers in which track segments are reconstructed, see Fig. 1. Down-sizing the number of used wires reduces largely the combinatorial problem but also reduces track information and redundancy.

The hit delays from the large drift volume and additional transmission delays leave only about $0.5 \mu \mathrm{s}$ for the actual track finding algorithms at L1. The high track multiplicity environment at HERA requires the reconstruction of up to 50 tracks/event. Both demands are tackled by using fast lookup techniques, which are typically three orders of magnitude faster than standard processor based Kalman filters. Lookup techniques implemented in a highly parallel design using CAMs are used for track segment finding (from hits) as well as for track linking.

High precision in track counting and track resolution is achieved by refining the lookup information in two stages. About $4 \times 3000$ drift time pattern templates are used to identify coarse track segments at L1. Up to $150 \times 100,000$ refined templates are used at L2. For each template the corresponding track parameter information is needed for track linking. In the L1 linking procedure this information is provided by hardwired register arrays with parallel access in one step. In the L2 linking procedure more precise track parameter information is provided by large associative memories with sequential access. High precision is achieved by using fully calibrated information based on the full 
offline reconstruction and by applying a primary vertex constraint.

Important issues, especially for a highly selective trigger as the FTT, are high track finding efficiency and large background rejection power. The latter might be compromised if wrong hit combinations or noise hits are linked and fake tracks are reconstructed. These effects are greatly reduced by requiring track segments to consist of three hits out of three wire layers. By using lookup techniques the combinatorial problem of hit assignment is solved already at the very first stage. Noise hits or tracks not originating from the primary interaction are reduced by demanding that the three hits and the primary vertex position can be fitted by a single trajectory. Redundancy, and consequently high track finding efficiency, is achieved by using relaxed criteria to link track segments to final tracks. The four trigger layers can provide up to four track segments per track but only two are required to form a track. This design ensures high track finding efficiency of $95 \%$ even if $5 \%$ of the hits are lost. Left-Right ambiguities which are a standard problem in track reconstruction are solved either at track segment finding level (not all possible solutions of three ambiguous hit positions can be extrapolated to the primary vertex) or eventually at track linking level.

\section{Hardware Implementation}

Front End System: The in total 30 Front End Modules (FEM) perform a fast digitization of the in total 900 input signals (450 wires, both ends) at $80 \mathrm{MHz}$. They also implement the digital processing including hit finding, charge integration, and track segment finding. One board processes information from five adjacent cells (per trigger layer) and provides all valid templates corresponding to track segments. This is done in five large FPGAs per board which implement embedded CAMs for fast lookup. Up to 3072 valid templates are stored per cell and trigger layer (trigger group).

L1 system: For each validated track segment the associated track information, i.e. the azimuthal angle $\Phi$ and the curvature $\kappa$ (transverse momentum), is sent to the L1 Linker. The L1 Linker is implemented on a single FTT Multipurpose Processing Board (MPB [2]) built by the SCS company [3]. It evaluates the $\kappa-\Phi$ information received from all trigger groups and layers using a $16 \times 60$ histogram. A peak finder searches for tracks by requiring a coincidence of track segments in two out of four trigger layers using a sliding window technique. The full algorithm is implemented in a single FPGA and performs the actual linking within 130 ns in a pipelined manner. Based on this information trigger decisions are made using track multiplicities, kinematical and topological information. 
L2 system: The larger latency allows the refinement of hit and track segment information and precise track fitting at L2. A single track segment template at L1 corresponds to 64 refined templates at L2 giving in total $\approx 200,000$ templates per trigger group. About $50 \%$ are validated when a precise hit matching of the three wires is required. This pre-calculated information stored in 4 MByte lookup memories on the FEM is sent sequentially to the L2 Linker. The L2 Linker uses a similar track finding algorithm as the L1 Linker. Because of the larger histogram $(40 \times 640$ bins $)$ its content is stored using an encoded format in CAMs which provide a fast linking of track segments. The track resolution at the linking step is about $10 \mathrm{mrad}$ in $\Phi$ and $30 \%$ in curvature for a $1 \mathrm{GeV} / \mathrm{c}$ track. These resolutions, mainly determining the track separation power, are further improved by about one order of magnitude by performing a vertex constrained fit in the $x-y$ plane. In addition, the polar angles of tracks are fitted in the $r-z$ plane. Both fits are implemented in a system of 24 DSPs based on six MPBs [2]. The main track parameter resolutions are $\sigma(\Phi)=2 \mathrm{mrad}, \sigma\left(1 / p_{T}\right)=2 \% /(\mathrm{GeV} / \mathrm{c})$, and $\sigma(\Theta) \approx 75 \mathrm{mrad}$, determined from 2005 data taking. The final L2 trigger decisions are based on criteria similar to L1 but with a much higher precision in momentum resolution.

L3 system: The L3 system consists of a farm of commercial VME Power PCs and runs VXWorks as real-time operating system. It implements timeoptimized routines to reconstruct event properties and decays of particle resonances from L2 tracks. It performs a trigger decision within $100 \mu \mathrm{s}$. The "show case" is the reconstruction of the "golden decay" of the $D^{*+}(2010)$ meson $\left(D^{*+} \rightarrow \pi^{+} K^{-} \pi_{s}^{+}\right)$. A first $D^{*+}$ mass peak as reconstructed by the FTT L2 in a commissioning run in 2005 is shown in Fig. 2.

\section{Conclusion and Outlook}

With the advent of large FPGAs with embedded CAMs and the construction of the FTT, a new era of online track triggering has begun at the H1 Experiment. The FTT allows for the first time a precise and complete reconstruction of tracks within $20 \mu$ s. High selectivity is achieved by implementing three trigger levels with increasing resolution and rejection power. At highest level multi-body decays are reconstructed and invariant mass combinations are calculated.

For future experiments these advanced, and still advancing, technologies might be considered to perform a full track reconstruction with high precision in even shorter processing times (latencies). Modern circuits like CAMs, mainly designed for high speed networking, could be considered as an interesting alternative to FPGA embedded CAMs, which are limited in memory size. 


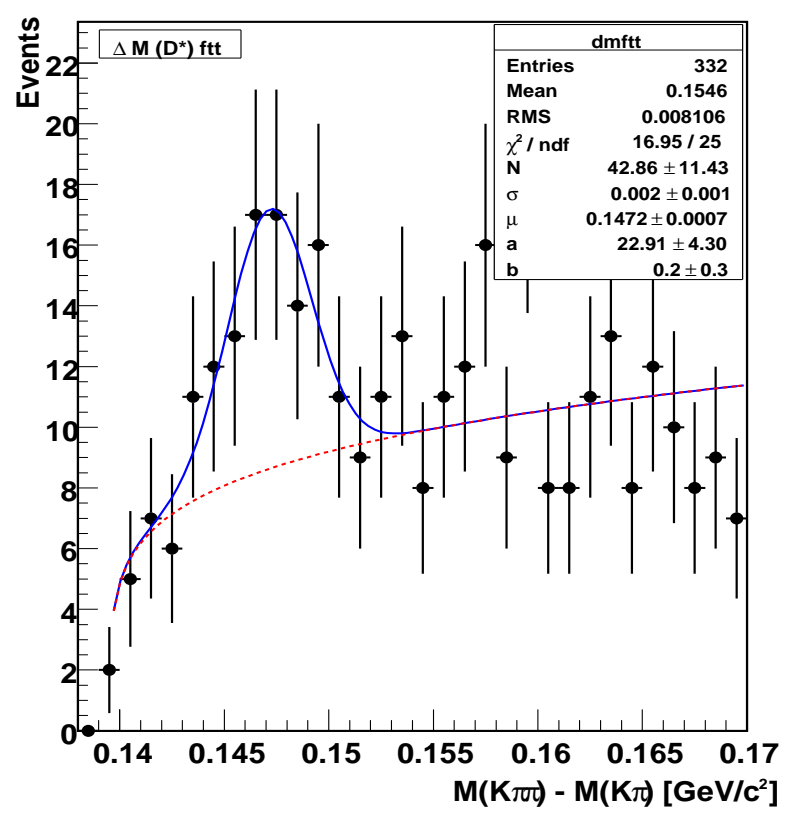

Fig. 2. Distribution of $\Delta M(K \pi \pi)-M(K \pi)$ for FTT reconstructed tracks at L2. A fit of the signal (blue line) and the background (red line) yields $42.9 \mathrm{D}^{*}$ candidates.

The most time consuming steps in track reconstruction, namely the hit finding, solving of the combinatorial problem and determination of the track parameters, can be very efficiently solved by highly parallel processing and lookup techniques implemented in hardware. Alternatively one could also consider the development of new processor types or processor boards which integrate many of the above features in a more general approach. Such systems could significantly speed up track reconstruction algorithms or other time consuming problems which are related to search tasks.

\section{Acknowledgment}

I would like to thank the organizers for their hospitality and initiative to organize this very successful workshop. I have to thank all members of the FTT group who turned this very ambitious and challenging project into reality.

\section{References}

[1] A. Baird et al., IEEE Trans. Nucl. Sci. 48 (2001) 1276 [arXiv:hep-ex/0104010].

[2] D. Meer et.al., IEEE Trans. Nucl. Sci. 49 (2002) 357 [arXiv:hep-ex/0107010].

[3] Supercomputing Systems, 8005 Zurich, Switzerland. [http://www.scs.ch] 